Metabolism

Manuscript Number: DIABET-D-16-00153R1

Title: Effectiveness of the multidisciplinary Risk Assessment and Management Program for patients with Diabetes Mellitus (RAMP-DM) on diabetic microvascular complications: a population-based cohort study

Article Type: Original Article

Keywords: diabetes mellitus, risk stratification, multidisciplinary, microvascular complications

Corresponding Author: Ms. Fangfang Jiao, MBBS

Corresponding Author's Institution: The University of Hong Kong

First Author: Fangfang Jiao, MBBS

Order of Authors: Fangfang Jiao, MBBS; Colman Siu Cheung Fung; Yuk Fai Wan; Sarah M McGhee; Carlos King Ho Wong; Daisy Dai; Ruby Kwok; Cindy Lo Kuen Lam

Manuscript Region of Origin: HONG KONG

Abstract: Aim: To evaluate the effectiveness of the multidisciplinary Risk Assessment and Management Program for Patients with Diabetes Mellitus (RAMP-DM) in reducing the risks of microvascular complications.

Methods: We conducted a prospective cohort study in 29,670 propensity score matched RAMP-DM participants and diabetic subjects under usual primary care $(14,835$ subjects in each group). The study endpoints were the first occurrence of any diabetic complications, nonproliferative diabetic retinopathy/pre-proliferative diabetic retinopathy (NPDR/prePDR), sight-threatening diabetic retinopathy (STDR) or blindness, nephropathy, end-stage renal disease (ESRD), neuropathy, and lower limb ulcer or amputation. Log rank-test and multivariable Cox proportional hazard regressions were employed to estimate the between-group differences in the incidences of study endpoints.

Results: After a median follow-up period of 36 months with >41,000 person-years, The RAMP-DM had lower incidence in any microvascular complications (760 versus 935, adjusted hazard ratio [HR],0.73; 95\% confidence interval [CI] $0.66-0.81 ; \mathrm{P}<0.001$ ). The RAMP-DM group had lower incidences in all the specific microvascular complications except neuropathy (adjusted HR, 0.94; 95\%CI, 0.61 - 1.45; P=0.778). The adjusted HR of the RAMP-DM to control group for ESRD, STDR or blindness, and lower limb ulcer or amputation were 0.40 (95\%CI, $0.24-0.69 ; \mathrm{P}<0.001), 0.55$ $(95 \% \mathrm{CI}, 0.39-0.78 ; \mathrm{P}=0.001)$, and $0.49(95 \% \mathrm{CI}, 0.30-0.80 ; \mathrm{P}=0.005)$, respectively.

Conclusion: The RAMP-DM intervention was associated with lower incidences of all microvascular complications except neuropathy over a three-year follow-up. The encouraging results provided evidence to support that 
structured risk assessment and risk-stratified management provided by a multidisciplinary team is effective in reducing microvascular complications in diabetic patients. 


\title{
Effectiveness of the multidisciplinary Risk Assessment and Management Program for patients with Diabetes Mellitus (RAMP-DM) on diabetic microvascular complications:
} a population-based cohort study

\section{Short title: Effects of RAMP-DM on microvascular complications}

Authors: Fangfang Jiao ${ }^{\mathrm{a}}$, Colman Siu Cheung Fung $^{\mathrm{a}}$, Yuk Fai Wan ${ }^{\mathrm{a}}$, Sarah Morag McGhee ${ }^{\mathrm{b}}$, Carlos King Ho Wong ${ }^{\mathrm{a}}$, Daisy Dai ${ }^{\mathrm{c}}$, Ruby Kwok ${ }^{\mathrm{c}}$, Cindy Lo Kuen Lam ${ }^{\mathrm{a}}$

\section{Corresponding author}

Fangfang Jiao

Email: francesj@connect.hku.hk

Telephone: 852-59382665

Fax: 852-28147475

Address: 3/F, Ap Lei Chau Clinic, 161 Ap Lei Chau Main Street, Ap Lei Chau, Hong Kong

Email addresses:

FJ: francesj@connect.hku.hk

CSCF:cfsc@hku.hk

YFW:yfwan@hku.hk

SMM: sarahmcghee7@gmail.com

CKHW: carlosho@hku.hk

DD: ddai@ha.org.hk

RK: kwoklp@ha.org.hk

CLKL: clklam@hku.hk

\begin{abstract}
Affiliations
a 3/F Ap Lei Chau Clinic, 161 Main Street, Ap Lei Chau, Hong Kong, Department of Family Medicine and Primary Care, Li Ka Shing Faculty of Medicine, The University of Hong Kong.
\end{abstract}


${ }^{\mathrm{b}}$ School of Public Health, Li Ka Shing Faculty of Medicine, The University of Hong Kong.5/F William MW Mong Block, 21 Sassoon Road, Hong Kong.

c Hospital Authority Building, 147B Argyle Street, Kowloon, Hong Kong, Primary and Community Services, Hospital Authority Head Office, Hong Kong Hospital Authority.

\section{Conflict of interest}

No conflicts of interest, financial or otherwise, are declared by the author(s).

\section{Disclosure statement}

There is nothing need to be disclosed. 
Methods: We conducted a prospective cohort study in 29,670 propensity score matched RAMPDM participants and diabetic subjects under usual primary care (14,835 subjects in each group). The study endpoints were the first occurrence of any diabetic complications, nonproliferative diabetic retinopathy/pre-proliferative diabetic retinopathy (NPDR/pre-PDR), sightthreatening diabetic retinopathy (STDR) or blindness, nephropathy, end-stage renal disease (ESRD), neuropathy, and lower limb ulcer or amputation. Log rank-test and multivariable Cox proportional hazard regressions were employed to estimate the between-group differences in the incidences of study endpoints.

Results: After a median follow-up period of 36 months with $>41,000$ person-years, The RAMPDM had lower incidence in any microvascular complications (760 versus 935, adjusted hazard ratio $[\mathrm{HR}], 0.73 ; 95 \%$ confidence interval $[\mathrm{CI}] \mathbf{0 . 6 6}-\mathbf{0 . 8 1} \boldsymbol{P}<\mathbf{0 . 0 0 1})$. The RAMP-DM group had lower incidences in all the specific microvascular complications except neuropathy (adjusted HR, 0.94; 95\%CI, 0.61 - 1.45; $P=0.778$ ). The adjusted HR of the RAMP-DM to control group for ESRD, STDR or blindness, and lower limb ulcer or amputation were 0.40 (95\% CI, 0.24 $0.69 ; P<0.001), 0.55$ (95\%CI, $0.39-0.78 ; P=0.001)$, and 0.49 (95\%CI, $0.30-0.80 ; P=0.005)$, respectively. 


\title{
Clinical trial registry: NCT02034695, www.ClinicalTrials.gov
}

Keywords: diabetes mellitus, risk stratification, multidisciplinary, microvascular complications

\author{
List of Abbreviations \\ DBP Diastolic Blood Pressure \\ DM Diabetes Mellitus \\ DR Diabetic Retinopathy \\ eGFR Estimated Glomerular Filtration Rate \\ ESRD End Stage Renal Disease \\ HR Hazard Ratio \\ ICD-9-CM International Classification of Diseases, Ninth Edition \\ ICPC-2 International Classification of Primary Care \\ NNT Number Needed to Treat \\ NPDR Non-Proliferative Diabetic Retinopathy \\ RAMP-DM Multidisciplinary Risk Assessment and Management Program for Patients with \\ diabetes mellitus \\ SBP Systolic Blood Pressure \\ STDR Sight-threatening Diabetic retinopathy
}

\section{Introduction}


Diabetes mellitus (DM) is one of the most common chronic diseases all over the world. The prevalence of DM in the world is estimated to reach 592 million by 2035, with an increase of $54.2 \%$ in total case numbers compared to the year of 2013[1]. China has the largest number of diabetic patients. The number of diabetic patients in China is estimated to exceed 129 million in 2030, accounting for more than one quarter of the total diabetic cases in the world [2]. In Hong Kong, around 1 in 10 people has DM [3], and the prevalence of DM is increasing [4]. Diabetic patients have an increased risk of developing microvascular complications, including retinopathy, nephropathy and neuropathy. The prevalence of retinopathy and neuropathy is estimated to be $22 \%$ and $13 \%$ respectively in newly diagnosed DM patients [5]. The prevalence of proliferative retinopathy is $2 \%$ in diabetic patients with less than 5 years of duration of $\mathrm{DM}$, and it increases $25 \%$ in patients with 25 or more years of DM [6]. Diabetic nephropathy develops in 25\% Type 2 DM patients after 10 years after diagnosis [7] and end-stage renal disease (ESRD) develops in 14\% Type 1 DM patients with 10 years duration of DM [8].

However, due to the insidious progress of microvascular complications, patients might be undetected or left untreated in early disease stage, which can lead to devastating impact on quality of life and life expectancy once the clinical significant complications are developed, such as ESRD, sight-threatening diabetic retinopathy (STDR), and amputation. Several modifiable risk factors, including HbA1c, blood pressure, eGFR are found to associate with development of microvascular complications [9-11]. Early screening and intervention of early stage of microvascular complications and modifiable risk factors are critical to prevent or delay the progress of disease severity. In recent years, guidelines have recommended risk factor screening and risk stratification management [12-14], setting personalized treatment goals based on patients' individual complication risks. Personalized management is advocated as a means of 
translating the evidence from randomized control trials to real-world settings [15]. However, there is a lack of studies on the effectiveness of risk stratification-based personalized management [15].

Previous studies on short-term effectiveness of risk-stratification based intervention were conducted in the U.S. [16] and U.K. [17] . Both studies reported the increase in the percentages of subjects reaching target $\mathrm{HbA1c}$, blood pressure in the intervention group. However, long-term effectiveness of the intervention was not reported. In Asia, attempt for the risk stratification management was made by the Joint Asia Diabetes Evaluation Program [18]. Clinicians can access a web-based comprehensive risk stratification model using an electronic portal. Between 2007-2009, 3687 people with diabetes across seven Asian countries, including Hong Kong, were enrolled [19]. The implementation of the structured care and effectiveness of this care model compared to usual care is not clear.

To enhance the management of diabetic subjects in primary care setting in Hong Kong, a multidisciplinary risk assessment and management program for patients with diabetes mellitus (RAMP-DM) has been operating in public general out-patient clinics since August 2009. The details of the intervention have been reported [20]. Compared to diabetic subjects under usual care, RAMP-DM group was found to have significant improvement in HbA1c, blood pressure control and reduction in cardiovascular events incidences at both 12-month [21] and 36-month [22] of follow-up. 


\section{Methods}

Study design

A prospective cohort study was conducted to compare the risks of developing different stages of three subtypes of diabetic microvascular complications over three years between diabetic subjects managed under RAMP-DM and those receiving usual primary care.

\section{RAMP-DM intervention}

The RAMP-DM was a territory-wide primary care service component for patients with DM in public primary care clinics in Hong Kong. It was launched since August 2009 by Hong Kong Hospital Authority, the sole public healthcare provider in Hong Kong. The details of the RAMPDM program have been reported previously [20]. In brief, all the enrolled subjects would undergo a comprehensive risk assessment examinations including measurement of basic parameters, laboratory test, eye and foot assessment. The case manager, taken by an advanced practice nurse would review the examinations results, assessed the cardiovascular risks and 


\section{Subjects}

The RAMP-DM aims at covering all diabetic patients in Hong Kong. All patients with DM who are followed up regularly at public primary care clinics are eligible to be enrolled in RAMP-DM. Patients were invited to join RAMP-DM opportunistically when they saw their primary care doctors. The enrollment was on a voluntary basis. Up to $31^{\text {st }}$ December 2013 , the end date of our study data collection period, there were 147,097 enrolled into RAMP-DM (out of a total of 206,238 patients receiving diabetic care under the primary care service of HA from August 2008 to July 2013). The remaining people with diabetes were continued to be enrolled into RAMP-DM after $31^{\text {st }}$ July 2013, and they served as potential control subjects in this study. 
We identified study subjects from the Clinical Management System database of the Hospital Authority. Inclusion criteria for this study are, 1) Age $\geq 18$; 2) Patients with documented International Classification of Primary Care (ICPC-2) codes T89/T90 before baseline; 3) Patients with at least one public primary clinics attendance before baseline. To evaluate the effectiveness of RAMP-DM in reducing primary microvascular complications, subjects with any pre-existing microvascular complications were excluded from the analysis. For the control group, patients who were enrolled in RAMP-DM on or before $31^{\text {st }}$ July 2013 were also excluded in the analysis.

The baseline for each RAMP-DM participant was the first date of risk assessment of RAMP-DM between $1^{\text {st }}$ August 2009 and $31^{\text {st }}$ July 2010. The subjects in control group were DM patients continuously managed in usual primary care. We set $31^{\text {st }}$ January 2010 , the middle date of the baseline among RAMP-DM subjects, as the baseline for the control group. All subjects were observed until a study endpoint or three years since their baseline using the date of their last follow-up as a censor date.

Ethical approval of this study was granted by the Institutional Review Board of the University of Hong Kong/Hospital Authority: Hong Kong West Cluster (UW 10-369), New Territories East Cluster (CRE-2010.543), New Territories West Cluster (NTWC/CREC/1091/12), Kowloon East and Kowloon Central Cluster (KC/KE-10-0210/ER-3), Kowloon West Cluster (KW/EX/10-317 (34-04)), and Hong Kong East Cluster (HKEC-2010-093).

Propensity score matching 
To reduce selection bias, the subjects in the RAMP-DM and control groups were matched using propensity score matching. Study subjects were paired based on observable characteristics which indicate a similar probability of receiving treatment (similar propensity score), but one of them received the intervention and the other did not. The propensity score is the conditional probability of receiving the intervention given the observed baseline covariates and it is independent of the outcomes. Propensity score matching is appropriate for studies with a large sample size and many covariates [23]. A propensity score was generated for each patient, and the RAMP-DM intervention was modelled as the dependent variable and the baseline covariates were the independent variables. The propensity score matching was conducted using the "psmatch2" STATA package by one-to-one matching without replacement and with a caliper of 0.001, which means the differences of propensity scores for each matched pair was within 0.001. The unmatched control subjects were discarded.

The baseline covariates for developing the propensity score were 1) demographic characteristics, including age, sex, whether on comprehensive social security assistance; 2) clinical parameters, including smoking status, duration of diabetes, HbA1c, low-density lipoprotein cholesterol (LDL-C), total cholesterol (TC), high-density lipoprotein cholesterol (HDL-C), triglyceride systolic blood pressure (SBP), diastolic blood pressure (DBP) and estimated glomerular filtration rate (eGFR); 3) treatment modality, including oral glucose-lowering drugs, insulin, antihypertensive drugs and lipid-lowering drugs; and 4) comorbidities, measured by the Charlson comorbidity score[24].

Study endpoints 


\section{Data analysis}

The independent t-test or chi-squared test, as appropriate, was employed to compare the demographic and clinical parameters between the RAMP-DM and control groups at baseline and the end of follow-up. The cumulative incidence rates for each subtype of microvascular complications were reported. We constructed the 95\% confidence intervals of the incidence rates based on the assumption that the observed incident events followed a Poisson distribution. We calculated the number needed to treat (NNT) to reduce one diabetesrelated complication by RAMP-DM. The NNT was calculated as the inverse of the absolute risk reduction [25]. The NNT is interpreted as the average number of patients needed to treat in order to reduce one unwanted outcomes. The lower the NTT, the more effective is the intervention.

For each of the study endpoints, the Kaplan-Meier method was used to estimate the survival curves and the log-rank test was used to compare the between group differences. To estimate the magnitude of differences in endpoints, multivariable Cox proportional hazards regression models were employed to explore the effects of RAMP-DM on the dependent variables of each endpoint, adjusting for all the baseline covariates. The hazard ratio (HR) with 95\% confidence interval of RAMP-DM were reported for each endpoint. The predictive accuracy 


\section{Results}

Baseline characteristics

From $1^{\text {st }}$ Aug 2009 to $31^{\text {st }}$ July 2010, a total of 18,459 diabetic subjects under primary care were enrolled in RAMP-DM. We identified 47,148 potential control subjects who met the inclusion criteria for the study. Subjects with any pre-existing microvascular complications were excluded in each group, giving 17,804 and 44,809 subjects in RAMP-DM and control groups, respectively. Limiting the eligibility to subjects with complete baseline data reduced the sample to 17,528 and 16,180 in RAMP-DM and control groups, respectively. To reduce selection bias, we further refined the study sample using propensity score matching. The final matched sample for this study comprised 14,835 RAMP-DM subjects and 14,835 control subjects.

The comparison of baseline characteristics between the two groups is shown in Table 1. At baseline, the RAMP-DM and control group had similar values in all the demographic, clinical parameters and treatment modality. The average age of the two cohorts was 65 , and around $87 \%$ subjects were on oral glucose-lowering drugs. At the end of follow-up, the RAMP-DM subjects 


\section{Observed incidence of microvascular complications}

Table 2 shows the observed number of the first diagnoses of each subtype of diabetic microvascular complication and the incidence rates over a median follow-up period of 36 months among 14,835 subjects in each group. More than 43,000 and 41,000 person-years of observation were available for each of the study endpoints in the RAMP-DM and control group, respectively. The RAMP-DM group showed lower incidence rates for all the endpoints. NPDR/non-PDR was the most prevalent microvascular complication in both groups (535 and 594 retinopathy events in RAMP-DM and control group, respectively). Over the observation period, 51 cases of STDR or blind occurred in the RAMP-DM group, which was around half that in the control group (90 cases). The number needed to treat by RAMP-DM to reduce one microvascular complication was 85 .

\section{Multivariable Cox Regression Models}

The Kaplan-Meier survival curves for each study end point are shown in Figure 1. For all severer microvascular complications, including STDR or blindness $(P<0.001)$, ESRD $(P<0.001)$, ulcer or amputation $(P=0.009)$, significant lower incidence rates were observed in RAMP-DM group, and the differences became larger over the follow-up period. The incidences of neuropathy were almost identical between the two groups $(P=0.778)$. 
The HR between RAMP-DM and control groups for each study endpoint was estimated by multivariable Cox regression models, adjusting for all the baseline covariates. As shown in Table 3, compared to the control group, the RAMP-DM group significantly reduced the incidence of both NPDR/pre-PDR (HR: 0.85, 95\% CI: 0.76-0.96, $P=0.007)$ and nephropathy (HR: 0.54, 95\% CI: 0.45-0.66, $P<0.001$ ). The reduction was more evident in severer stages of disease. The HR for STDR or blind and ESRD were 0.55 (95\%CI: $0.39-0.78, P=0.001)$ and $0.40(95 \% C I: 0.24-$ 0.69, $P<\mathbf{0 . 0 0 1}$ ), respectively. The two groups did not show difference in the incidence of neuropathy (HR: 0.94, 95\% CI: 0.61-1.45, $P=\mathbf{0 . 7 8}$ ), but the RAMP-DM group had substantial lower incidence in ulcer or amputation (HR: 0.49, 95\% CI: 0.30-0.80, P=0.005).

\section{Discussion}

To the best of our knowledge, this was the first study investigating the long-term effects of a multidisciplinary risk-stratification based DM management on microvascular complications in Chinese population. With more than 41,000 person-years follow-up, this population-based cohort study found that RAMP-DM intervention was associated with reduction in the incidences of all the studied diabetic microvascular complications, except neuropathy. The RAMP-DM was more effective in reducing the incidences of severe microvascular complications, prompting that the HR for developing STDR or blindness, ESRD and ulcer or amputation were $\mathbf{0 . 5 5 , 0 . 4 0}$ and 0.49, respectively. These findings provided evidence of the effectiveness of multidisciplinary risk-stratification based management in a real world primary setting. 
Significant lower incidences of retinopathy, nephropathy and amputation were observed in the RAMP-DM group, which might be partly attributed to the significant decreases in HbA1c and blood pressure observed in the RAMP-DM group. Also, the RAMP-DM group had higher percentage of subjects on glucose-lowering drugs, insulin, anti-hypertensive drugs and lipidlowering drugs, indicating the RAMP-DM participants were under more intensive treatment. HbA1c and blood pressure were found to be associated with the risks of developing diabetic retinopathy [9], nephropathy [10] and neuropathy [27] . Previous studies found that intensive glucose control were associated with lower incidences of microvascular complications. The United Kingdom Prospective Diabetes Study found that, over 10 years follow-up, the intensive glucose control reduced the risk of aggregated microvascular complications (RR: $0.75, P=0.0099$ ) and retina photocoagulation (RR: $0.71, P=0.0031$ ), but no effects were found in reducing renal failure (RR: 0.73, $P=0.45$ ) and amputation (RR: 0.61, $P=0.099$ ) [28]. The Preterax and Diamicron Modified Release Controlled Evaluation trial showed that over 5 years of follow-up, the intensive control group showed decrease in the incidence of nephropathy (HR 0.79, $P=0.006$ ), but not significant effect on retinopathy (HR 0.95, $P=0.50$ ) [29]. Compared to interventions that only emphasized on medical intervention, multidisciplinary interventions were found to be more effective to reduce renal and ophthalmological complications. The Steno-2 study implemented a multifactorial intervention including a combination of medications and focused behavior modification [30]. It was reported that the intervention group had lower risks for nephropathy (relative risk $0.44, P=0.004$ ), retinopathy (relative risk $0.57, P=0.01$ ) and autonomic neuropathy (relative risk $0.53, P=0.004$ ) over 13.3 years follow-up compared to the conventional care group . A physician-led structured diabetes management program in Germany also showed lower incidence of chronic renal insufficiency (relative risk 0.49) and amputation (relative risk 0.63) 

For each subtype of microvascular complication, we investigated both early stage and advanced
stage of disease. Interestingly, we found RAMP-DM was more effective in reducing more severe
stage of disease. RAMP-DM group showed substantial decrease in the incidence of STDR or
blindness (HR: $0.55, \mathrm{P}=0.001$ ), while for the incidence of mild diabetic retinopathy (NPDR/prePDR), the difference between two groups was not evident until 24 months (Figure 1). A possible explanation might be that diabetic subjects under RAMP-DM had systematic diabetic retinopathy screening, leading to a higher detection rate of diabetic retinopathy. Early detection allows more timely management for subjects diagnosed with mild, non-clinical significant diabetic retinopathy which might result in a lower incidence of STDR. As diabetic retinopathy can be totally asymptomatic until it develops into STDR, early screening for diabetic retinopathy is critical in preventing further deterioration by giving timely intensive treatment. A previous study showed that a systematic screening program could effectively reduce the prevalence of blindness [32]. We should note that even the RAMP-DM group might have higher detect rate for NPDR/non-PDR due to the systemic screening, the RAMP-DM management was still associated with significant lower incidences of NPDR/non-PDR, which might due to better control of blood glucose and blood pressure.

The similar findings were observed for neuropathy. The incidence of mild neuropathy (lower limb ulcer or amputation not included) was similar between RAMP-DM and control groups (HR: 0.940, $\mathrm{P}=0.778$ ), and the survival curves were almost overlapped (Figure 1). However, RAMP- 


\begin{abstract}
DM group had significantly lower incidence of lower limb ulcer or amputation (HR: 0.493,
\end{abstract} $\mathrm{P}=0.005$ ), and the difference became more evident after 12 months. Patients enrolled in RAMPDM undertook comprehensive foot examination, including physical examination for callosity, corn, ulcer, skin infection, deformities, nail pathology, temperature, ischaemic changes and peripheral pulses, monofilament test and vibration perception threshold by biothesiometer. Early screening was likely to detect more cases asymptomatic neuropathy, leading to relatively "higher" incidence of early stage neuropathy in the RAMP-DM group. While the incidence of lower limb ulcer or amputation was remarkably lower in the RAMP-DM group. Tight glycemic control [27, 33], structured education program [34] and multidisciplinary management [30, 31] have been found in other studies to decrease the incidence of neuropathy effectively. As a multidisciplinary intervention, RAMP-DM included disease education on knowledge, self-care and lifestyle during nurse intervention. The risk-stratification management might also raise the doctors' awareness to offer more intensive management of high risk patients. All these efforts might contribute to the lower incidences of severer neuropathy in the RAMP-DM group.

We found RAMP-DM was associated with lower incidences of both nephropathy (ESRD not included) and ESRD. The presence of asymptomatic nephropathy was mainly through albuminuria and eGFR. Diabetic patients under usual primary care also undertook annual blood test in Hong Kong. However, patients enrolled in RAMP-DM might receive more intensive management in preventing future deterioration of disease, resulting in lower incidences of nephropathy and ESRD. 
RAMP-DM is the program to stratify diabetic patients with different risk levels so that they can receive regular assessment and screening and can be provided early with additional interventions or management. RAMP-DM is a way of increasing the focus on patients that need a more intensive treatment. The RAMP-DM is organizational investment from the Hospital Authority and Food \& Health Bureau, that administrative support, central information technology system and screening facilities were well provided. In addition, the Food \& Health Bureau of government commissioned annual audit and evaluation of quality of care in three consecutive years, which facilitate quality improvement of diabetes care and adherence to the structured risk-stratification based protocol among RAMP-DM patients. Therefore, this program could effectively translate the systematic management protocol into the real-world setting.

\section{Strength and limitations of this study}

This propensity score matched prospective comparative effectiveness study had several strengths. First, the study sample was extracted from the Clinical Management Systemof the Hospital Authority, which recorded data on all the people with diabetes managed in the public healthcare sector. This population-based sample was highly representative of the Hong Kong population with diabetes. Second, large sample size and three-year follow-up presented sufficient subjects to examine different stage of diabetic microvascular complications. Third, comprehensive covariates were included to develop propensity score matching for the two groups. The observed risk factors that might affect the incidence of diabetic complications were included. We further adjusted all the covariates during multivariable Cox regression to minimize any possible bias. Fourth, this comparative effectiveness study was based on data obtained from real patients under 
primary care, and thus applicable to a real-world setting. Fifth, we used an intention-to-treat analysis, giving a more conservative estimate of the effectiveness of RAMP-DM.

Several limitations should be aware when interpreting the results. The important limitation of this study was that we could not carry out a randomized study therefore unobserved potential confounders might affect the results, although we have minimised this by including as many possible covariates as we can in propensity score matching. Second, not all the RAMP-DM subjects were included in the analysis due to missing data at baseline and some subjects were further excluded due to unavailable matched control pairs. Third, the RAMP-DM patients and usual care patients might come from the same or different public primary care clinics. Unfortunately, we did not have the clinic information since all the patients were free to choose different public primary care clinics for their follow-up. Fourth, three years are not long enough to project the long term benefits of RAMP-DM. We need longer follow-up to evaluate the longer term effects of RAMP-DM. It would be interesting to see whether the effects of RAMP-DM would maintain over a longer term.

\section{Conclusions}

This prospective comparative effectiveness study in a pragmatic primary care setting found that RAMP-DM was associated with decreased risks of first occurrence of retinopathy, STDR, nephropathy, ESRD, and lower limb ulcer or amputation over a three-year follow-up. These findings supplemented the effectiveness of RAMP-DM and provided imperative translational evidence of the effectiveness of multidisciplinary risk-stratification based management for people with diabetes. 


\section{Acknowledgements}

This Study on Enhanced Primary Care was funded by the Food and Health Bureau, the Government of the Hong Kong Special Administrative Region (Project No.EPC-HKU-2). The authors wish to acknowledge the contributions of the RAMP-DM program teams (including Ms. Dorothy Lam, Mr. Jackey Chan) and Statistics and Workforce Planning Department (including Ms. Eva Tsui, Mr. Peggo Lam, Mr. Choi-Fan Yiu) at the Hong Kong Hospital Authority. Also, we would like to thank all hospital authority cluster coordinators and clinical staff in the Chronic Disease Management Programs for working with our team in this evaluation study.

\section{References}

[1] Guariguata L, Whiting D, Hambleton I, Beagley J, Linnenkamp U, Shaw J. Global estimates of diabetes prevalence for 2013 and projections for 2035. Diabetes Res Clin Pr. 2014;103(2):137-49.

[2] International Diabetes Federation. Diabetes Atlas. sixth edition. 2013.

[3] Janus E, Wat N, Lam K, Cockram C, Siu S, Liu L, et al. The prevalence of diabetes, association with cardiovascular risk factors and implications of diagnostic criteria (ADA 1997 and WHO 1998) in a 1996 community - based population study in Hong Kong Chinese. Diabetic Med. 2000;17(10):741-5.

[4] Wong KC, Wang Z. Prevalence of type 2 diabetes mellitus of Chinese populations in Mainland China, Hong Kong, and Taiwan. Diabetes Res Clin Pr. 2006;73(2):126-34.

[5] Leung G, Lam K. Diabetic complications and their implications on health care in Asia. Hong Kong medical journal= Xianggang yi xue za zhi/Hong Kong Academy of Medicine. 2000;6(1):61-8.

[6] Klein R, Klein BE, Moss SE, Davis MD, DeMets DL. The Wisconsin Epidemiologic Study of Diabetic Retinopathy: II. Prevalence and risk of diabetic retinopathy when age at diagnosis is less than 30 years. Arch Ophthalmol-chic. 1984;102(4):520-6.

[7] Adler AI, Stevens RJ, Manley SE, Bilous RW, Cull CA, Holman RR. Development and progression of nephropathy in type 2 diabetes: the United Kingdom Prospective Diabetes Study (UKPDS 64). Kidney Int. 2003;63(1):225-32. 
[8] Brancati FL, Whelton PK, Randall BL, Neaton JD, Stamler J, Klag MJ. Risk of end-stage renal disease in diabetes mellitus: a prospective cohort study of men screened for MRFIT. JAMA. 1997;278(23):2069-74.

[9] Aspelund T, pórisdóttir Ó, Olafsdottir E, Gudmundsdottir A, Einarsdóttir A, Mehlsen J, et al. Individual risk assessment and information technology to optimise screening frequency for diabetic retinopathy. Diabetologia. 2011;54(10):2525-32.

[10] Jardine MJ, Hata J, Woodward M, Perkovic V, Ninomiya T, Arima H, et al. Prediction of kidney-related outcomes in patients with type 2 diabetes. Am J Kidney Dis. 2012;60(5):770-8.

[11] Girach A, Manner D, Porta M. Diabetic microvascular complications: can patients at risk be identified? A review. Int J Clin Pract. 2006;60(11):1471-83.

[12] American Diabetes Association. Standards of Medical Care in Diabetes 2013. Diabetes Care. 2013;36(Supplement 1):S11-S57.

[13] National Collaborating Centre for Chronic Conditions. Type 2 diabetes National clinical guideline for management in primary and secondary care (update). Royal College of physicians, 2008.

[14] Canadian Diabetes Association. 2008 Clinical Practice Guidelines for the Prevention and Management of Diabetes in Canada. Can J Diabetes 2008.32(supplement 1):S1-S201.

[15] Esposito K, Gentile S, Candido R, De Micheli A, Gallo M, Medea G, et al. Management of hyperglycemia in type 2 diabetes: evidence and uncertainty. Cardiovas diabetol. 2013;12(1):81.

[16] Clark CM, Snyder JW, Meek RL, Stutz LM, Parkin CG. A systematic approach to risk stratification and intervention within a managed care environment improves diabetes outcomes and patient satisfaction. Diabetes Care. 2001;24(6):1079-86.

[17] Harris M, Greaves F, Patterson S, Jones J, Pappas Y, Majeed A, et al. The North West London Integrated Care Pilot: innovative strategies to improve care coordination for older adults and people with diabetes. J Ambul Care Manage. 2012;35(3):216-25.

[18] Chan J, So W, Ko G, Tong P, Yang X, Ma R, et al. The Joint Asia Diabetes Evaluation (JADE) Program: a web - based program to translate evidence to clinical practice in Type 2 diabetes. Diabetic Med. 2009;26(7):693-9.

[19] So WY, Raboca J, Sobrepena L, Yoon KH, Deerochanawong C, Ho LT, et al. Comprehensive risk assessments of diabetic patients from seven Asian countries: The Joint Asia Diabetes Evaluation (JADE) program*. Journal of diabetes. 2011;3(2):109-18.

[20] Fung CS, Chin WY, Dai DS, Kwok RL, Tsui EL, Wan YF, et al. Evaluation of the quality of care of a multi-disciplinary risk factor assessment and management programme (RAMP) for diabetic patients. BMC Fam Pract. 2012;13:116.

[21] Jiao FF, Fung CS, Wong CK, Wan YF, Dai D, Kwok R, et al. Effects of the Multidisciplinary Risk Assessment and Management Program for Patients with Diabetes Mellitus (RAMP-DM) on biomedical outcomes, observed cardiovascular events and cardiovascular risks in primary care: a longitudinal comparative study. Cardiovas diabetol. 2014;13(1):127.

[22] Jiao F, Fung CS, Wan YF, McGhee SM, Wong CK, Dai D, et al. Long-term effects of the multidisciplinary risk assessment and management program for patients with diabetes mellitus (RAMP-DM): a population-based cohort study. Cardiovas Diabetol. 2015;14(1):105.

[23] Dehejia RH, Wahba S. Propensity score-matching methods for nonexperimental causal studies. Rev Econ Stat. 2002;84(1):151-61. 
[24] Charlson M, Peterson J, Szatrowski TP, MacKenzie R, Gold J. Long-term prognosis after peri-operative cardiac complications. J Clin Epidemiol. 1994;47(12):1389-400.

[25] Laupacis A, Sackett DL, Roberts RS. An assessment of clinically useful measures of the consequences of treatment. New Engl J Med. 1988;318(26):1728.

[26] Antolini L, Boracchi P, Biganzoli E. A time - dependent discrimination index for survival data. Stat Med. 2005;24(24):3927-44.

[27] Tesfaye S, Chaturvedi N, Eaton SE, Ward JD, Manes C, Ionescu-Tirgoviste C, et al. Vascular risk factors and diabetic neuropathy. New Engl J Med. 2005;352(4):341-50.

[28] Group UPDS. Intensive blood-glucose control with sulphonylureas or insulin compared with conventional treatment and risk of complications in patients with type 2 diabetes (UKPDS 33). The Lancet. 1998;352(9131):837-53.

[29] Patel A, MacMahon S, Chalmers J, Neal B, Billot L, Woodward M, et al. Intensive blood glucose control and vascular outcomes in patients with type 2 diabetes. New Engl J Med. 2008;358(24):2560-72.

[30] Gæde P, Lund-Andersen H, Parving H-H, Pedersen O. Effect of a multifactorial intervention on mortality in type 2 diabetes. New Engl J Med. 2008;358(6):580-91.

[31] Stock S, Drabik A, Büscher G, Graf C, Ullrich W, Gerber A, et al. German diabetes management programs improve quality of care and curb costs. Health Affair. 2010;29(12):2197205.

[32] Stefánsson E, Bek T, Porta M, Larsen N, Kristinsson JK, Agardh E. Screening and prevention of diabetic blindness. Acta Ophthalmol Scan. 2000;78(4):374-85.

[33] Holman RR, Paul SK, Bethel MA, Matthews DR, Neil HAW. 10-year follow-up of intensive glucose control in type 2 diabetes. New Engl J Med. 2008;359(15):1577-89.

[34] Wong CK, Wong WC, Wan Y, Chan AK, Chan FW, Lam CL. Patient Empowerment Programme (PEP) and Risk of Microvascular Diseases Among Patients With Type 2 Diabetes in Primary Care: A Population-Based Propensity-Matched Cohort Study. Diabetes Care. 2015;38(8):e116-e7. 
Figure legends

Figure 1. Kaplan-Meier survival curves

ESRD, End stage renal disease; STDR, Sight-threatening diabetic retinopathy.

All the P values were from Log-rank test. 


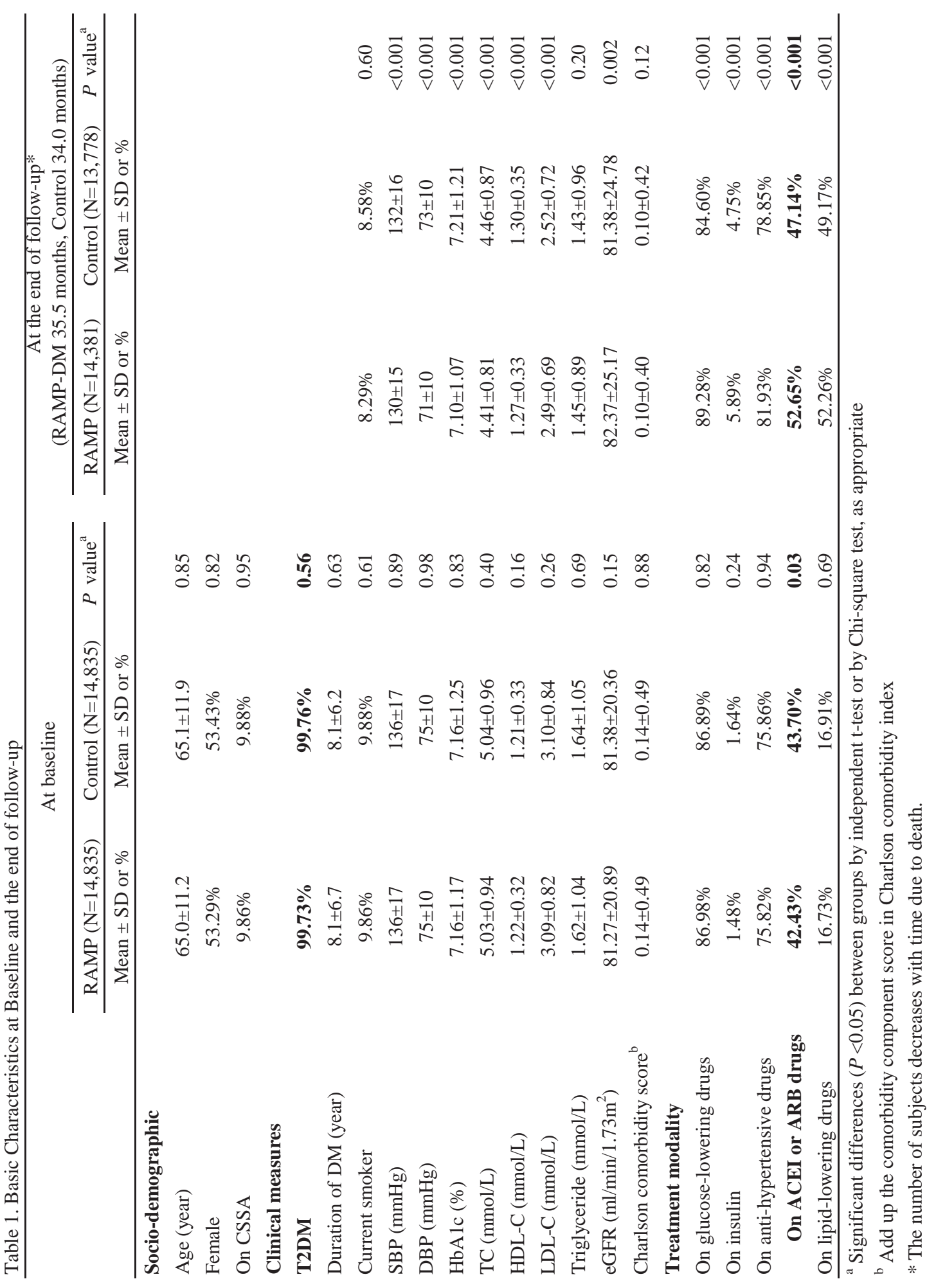




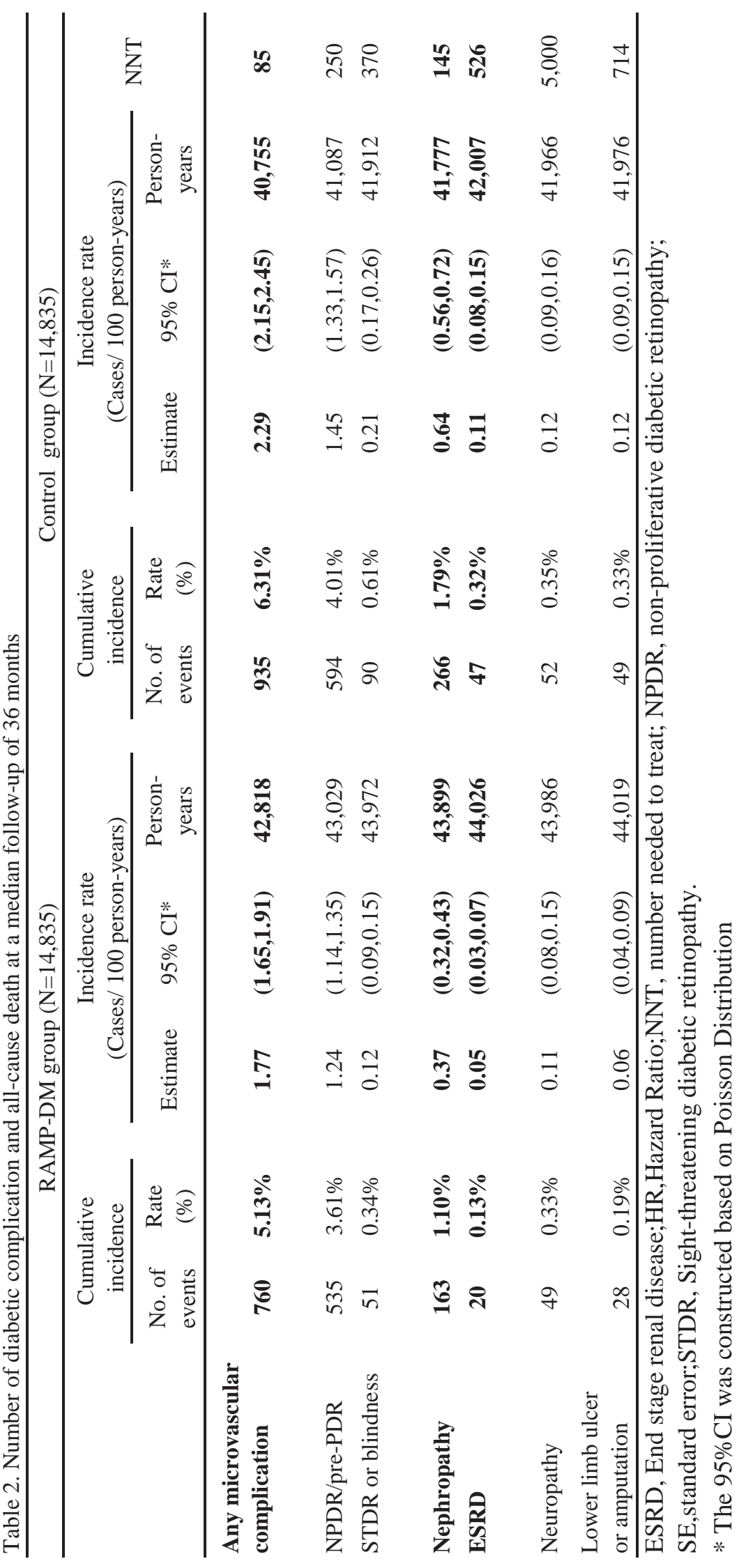


Table 3. Multivariable Cox proportional hazard regression all endpoints

\begin{tabular}{lcccc}
\hline & \multicolumn{4}{c}{ RAMP-DM vs Control } \\
\cline { 2 - 5 } & HR $\dagger$ & SE & 95\%CI & P-value \\
\hline RAMP-DM subjects vs Control subjects (All subjects, & $\mathbf{N}=\mathbf{2 9 , 6 7 0 )}$ & \\
Any microvascular complications & $\mathbf{0 . 7 3}$ & $\mathbf{0 . 0 4}$ & $\mathbf{( 0 . 6 6 , 0 . 8 1 )}$ & $<\mathbf{0 . 0 0 1}$ \\
& & & & \\
NPDR/pre-PDR & 0.85 & 0.05 & $(0.76,0.96)$ & 0.01 \\
STDR or blindness & 0.55 & 0.10 & $(0.39,0.78)$ & 0.001 \\
& & & & \\
Nephropathy & $\mathbf{0 . 5 4}$ & $\mathbf{0 . 0 5}$ & $\mathbf{( 0 . 4 5 , 0 . 6 6 )}$ & $<\mathbf{0 . 0 0 1}$ \\
ESRD & $\mathbf{0 . 4 0}$ & $\mathbf{0 . 1 1}$ & $\mathbf{( 0 . 2 4 , 0 . 6 9 )}$ & $<\mathbf{0 . 0 0 1}$ \\
& & & & \\
Neuropathy & 0.94 & 0.21 & $(0.61,1.45)$ & 0.78 \\
Ulcer or amputation & 0.49 & 0.12 & $(0.30,0.80)$ & 0.01 \\
\hline
\end{tabular}

ESRD, End stage renal disease;HR=Hazard Ratio;NPDR, non-proliferative diabetic reti $\mathrm{SE}=$ standard error;STDR, Sight-threatening diabetic retinopathy.

$\dagger \mathrm{HR}>1$ indicates greater risk for endpoints

Adjusted for age, sex, whether on CSSA, duration of DM, smoking status, SBP, DBP, HbA1c, TC, ] triglyceride, eGFR, Charlson comorbidity score, glubose-lowering drugs, insulin, anti-hypertensive drugs and lipid-lowering drugs. 


\section{Supplementary Table}

Supplementary Table. ICD-9CM, ICPC-2 codes for diabetic macrovas Disease ICPC-2 Codes

Nonproliferative diabetic retinopathy

F83

/pre-proliferative diabetic retinopathy

F94

or blindness

NA

Diabetic nephropathy

NA

Neuropathy

N94

Ulcer of lower limb or amputation

L81 
Click here to download high resolution image
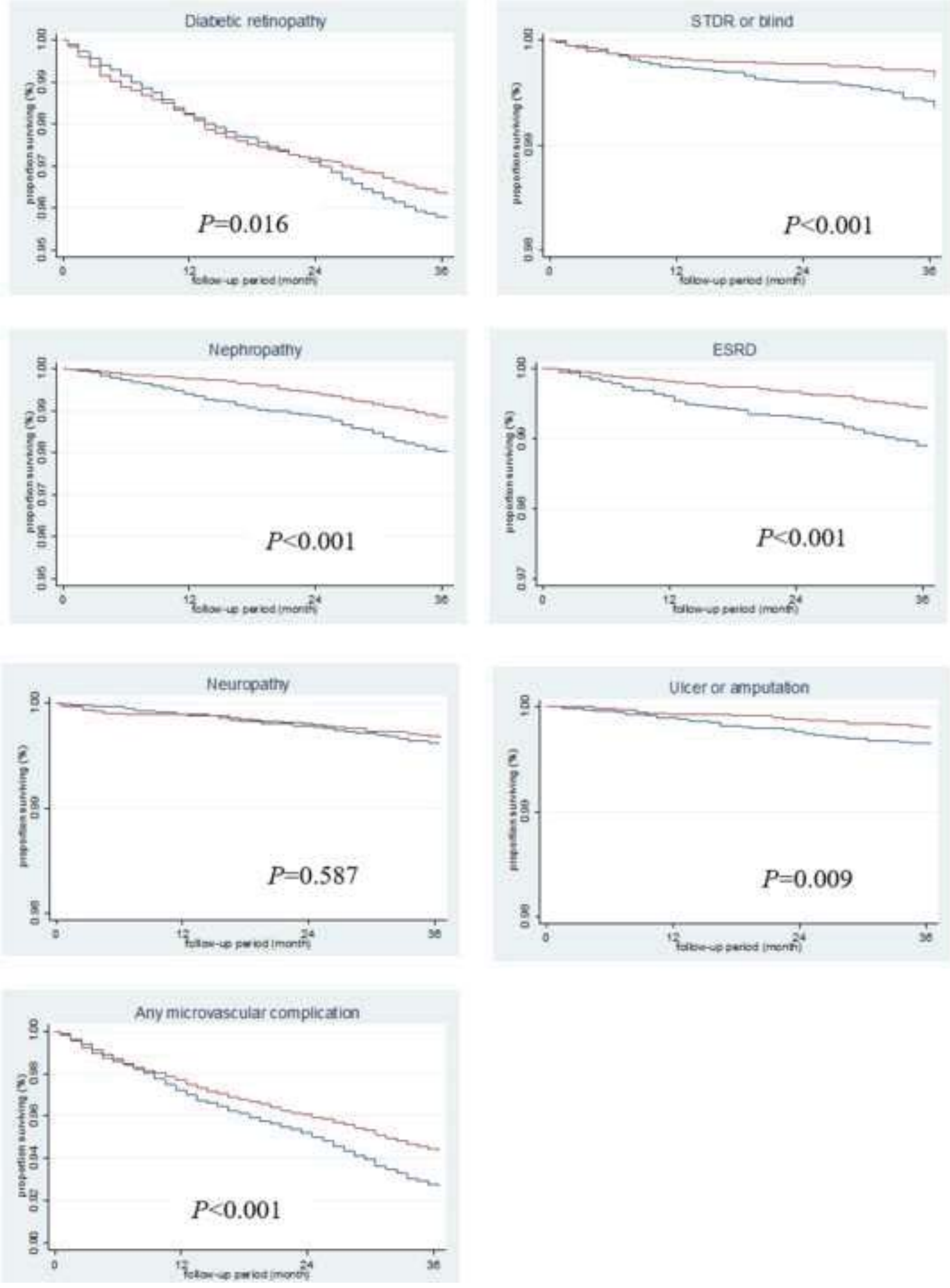